\title{
EVIDENCE FOR LOW EXTINCTION IN ACTIVELY STAR-FORMING GALAXIES AT $z>6.5$
}

\author{
F. Walter ${ }^{1}$, R. Decarli ${ }^{1}$, C. Carilli ${ }^{2}$, D. Riechers ${ }^{3}$, F. Bertoldi $^{4}$, A. Wei $\beta^{5}$, P. Cox $^{6}$, R. Neri $^{6}$, \\ R. MAIOLINO ${ }^{7}$, M. OUChI ${ }^{8}$, E. Egami ${ }^{9}$, AND K. NAKANishi ${ }^{10,11,12}$ \\ ${ }^{1}$ Max-Planck-Institut für Astronomie, Königstuhl 17, D-69117 Heidelberg, Germany; walter@mpia.de \\ ${ }^{2}$ National Radio Astronomy Observatory, 1003 Lopezville Road, Socorro, NM 87801, USA \\ ${ }^{3}$ Astronomy Department, California Institute of Technology, 1200 East California Blvd., Pasadena, CA 91125, USA \\ ${ }^{4}$ Argelander-Institut für Astronomie, Auf dem Hügel 71, D-53121 Bonn, Germany \\ ${ }^{5}$ Max-Planck-Insitut für Radioastronomie, Auf dem Hügel 69, D-53121 Bonn, Germany \\ ${ }^{6}$ Institut de Radio Astronomie Millimétrique (IRAM), F-38406, St. Martin d'Hères, France \\ ${ }^{7}$ Institute of Astronomy, Cavendish Laboratory, University of Cambridge, 19 JJ Thomson Avenue, Cambridge CB3 0HE, UK \\ ${ }^{8}$ Observational Cosmology Group, University of Tokyo, ICRR, 5-1-5 Kashiwanoha, Kashiwa 277-0882, Japan \\ ${ }^{9}$ Department of Astronomy/Steward Observatory, University of Arizona, 933 North Cherry Avenue, Tucson, AZ 85721-0065, USA \\ ${ }^{10}$ ALMA Project Office, National Astronomical Observatory, 2-21-1 Osawa, Mitaka, Tokyo 181-8588, Japan \\ ${ }^{11}$ Joint ALMA Office, Alonso de Cordova 3107, Vitacura, Santiago 763 0355, Chile \\ 12 The Graduate University for Advanced Studies (Sokendai), 2-21-1 Osawa, Mitaka, Tokyo 181-8588, Japan \\ Received 2011 November 18; accepted 2012 April 13; published 2012 May 30
}

\begin{abstract}
We present a search for the [C II] $158 \mu \mathrm{m}$ fine structure line (a main cooling line of the interstellar medium) and the underlying far-infrared (FIR) continuum in three high-redshift $(6.6<z<8.2)$ star-forming galaxies using the IRAM Plateau de Bure Interferometer. We targeted two Ly $\alpha$-selected galaxies (Ly $\alpha$ emitters, LAEs) with moderate UV-based star formation rates (SFRs $\sim 20 M_{\odot} \mathrm{yr}^{-1}$; Himiko at $z=6.6$ and $I O K-1$ at $z=7.0$ ) and a gamma-ray burst (GRB) host galaxy (GRB 090423 at $z \sim 8.2$ ). Based on our $3 \sigma$ rest-frame FIR continuum limits, previous (rest-frame) UV continuum measurements and spectral energy distribution (SED) fitting, we rule out SED shapes similar to highly obscured galaxies (e.g., Arp 220, M 82) and less extreme dust-rich nearby spiral galaxies (e.g., M 51) for the LAEs. Conservatively assuming an SED shape typical of local spiral galaxies we derive upper limits for the FIR-based star formation rates (SFRs) of $\sim 70 M_{\odot} \mathrm{yr}^{-1}, \sim 50 M_{\odot} \mathrm{yr}^{-1}$, and $\sim 40 M_{\odot} \mathrm{yr}^{-1}$ for Himiko, IOK-1, and GRB 090423, respectively. For the LAEs these limits are only a factor $\sim 3$ higher than the published UV-based SFRs (uncorrected for extinction). This indicates that the dust obscuration in the $z>6$ LAEs studied here is lower by a factor of a few than what has recently been found in some LAEs at lower redshift $(2<z<3.5)$ with similar UV-based SFRs. A low obscuration in our $z>6$ LAE sample is consistent with recent rest-frame UV studies of $z \sim 7$ Lyman break galaxies.
\end{abstract}

Key words: cosmology: observations - galaxies: evolution - galaxies: formation - infrared: galaxies

\section{INTRODUCTION}

Characterizing the physical properties of the earliest galaxies in the universe is a key goal in observational astrophysics. Of particular interest is the redshift range $z>6$ (corresponding to the first Gyr of the universe), in which the universe underwent a phase transition from a mostly neutral to a mostly ionized universe (the "Epoch of Reionization," see, e.g., reviews by Fan et al. 2006 and Robertson et al. 2010). Characterizing the physical properties of galaxy populations in this early epoch and their respective contribution to reionization are important drivers in current studies of the high-redshift universe. Recent deep dropout studies in the Hubble Ultra Deep Field using the Hubble Space Telescope have revealed a population of $z>6.5$ galaxy candidates, out to possibly $z \sim 10$ (e.g., Bouwens et al. 2011). Given their faintness in optical and near-infrared (NIR) bands, spectroscopic confirmation of these sources has been challenging, leading to only a few confirmed galaxies at $z \gtrsim 7.0$ (e.g., Fontana et al. 2010; Vanzella et al. 2011; Kashikawa et al. 2011; Ono et al. 2012; Pentericci et al. 2011; Schenker \& Stark 2012).

Galaxies with the highest Ly $\alpha$-based SFRs at these redshifts $(z>6.5)$ are selected through wide-field, narrowband surveys, the so-called Ly $\alpha$ emitters (hereafter LAEs). A high fraction of narrowband-selected candidates is spectroscopically confirmed to indeed lie at $z>6.5$ (e.g., Taniguchi et al. 2005; Iye et al. 2006; Ouchi et al. 2009, 2010; Kashikawa et al. 2011; Jiang et al.
2011). Their Ly $\alpha$ fluxes imply SFRs of typically $\sim 10 M_{\odot} \mathrm{yr}^{-1}$, consistent with their (rest-frame) UV luminosities. A recent study of low- $z(z \sim 0.3)$ LAEs by Oteo et al. (2012a) with lower UV-based SFRs finds that obscured star formation contributes to more than $50 \%$ of the total SFR, as traced by both UV and FIR emission. In a higher redshift $(2.0<z<3.5)$ sample of LAEs, Oteo et al. (2012b) report that some FIR-based SFRs (for objects with UV-based SFRs comparable to the sources studied here) contribute more than $90 \%$ of the total SFRs (their Table 1). This implies that the presence of detectable dust and Ly $\alpha$ emission is not mutually exclusive in these objects (Oteo et al. 2012b).

An alternative way to pinpoint the locations of active star formation in the very early universe is by gamma-ray bursts (GRBs) that are thought to be a common phenomenon at redshifts $z>6.5$ (e.g., review by Woosley \& Bloom 2006; Bromm \& Loeb 2006; Salvaterra \& Chincarini 2007). Observations indicate that the GRB rate may evolve even more rapidly than the star formation history of the universe up to $z=4$ (Kistler et al. 2008; Yüksel et al. 2008). Since the progenitors of long GRBs are thought to be short-lived massive stars of one of the universe's earliest stellar generation, their detection at extreme redshifts offers the possibility to put approximate constraints on the star formation rates (SFRs) in their host galaxies.

Although $z>6.5$ galaxies are key to understanding early galaxy formation and reionization, it is a major challenge to study them at their extreme redshifts. Even with long (tens 
of hours) exposures on 8-10 m class telescopes, optical/NIR observations can only detect the Ly $\alpha$ line and the underlying UV stellar continuum. Indeed, essentially all of our current knowledge on these reionization sources is based on the highly resonant Ly $\alpha$ line and the faint underlying continuum, which is potentially affected by dust attenuation, and generally difficult to interpret due to the complexity of the $\operatorname{Ly} \alpha$ radiative transfer.

Observations of the (sub-)millimeter continuum and spectral lines in principle provide the means by which to constrain basic physical parameters of the highest redshift sources (e.g., size, mass, distribution of the star-forming interstellar medium (ISM) and star formation, as well as dynamical masses). Traditionally, $\mathrm{CO}$ emission lines are used to characterize the molecular reservoirs in high-redshift galaxies (e.g., reviews by Solomon \& Vanden Bout 2005; Walter et al. 2011). However, the expected $\mathrm{CO}$ line strengths even in the brightest $z>6.5$ (non-AGN) galaxies are $\sim$ tens of $\mu \mathrm{Jy}-$ such faint lines are not accessible given current facilities and will even be a challenge with ALMA. On the other hand, the ${ }^{2} P_{3 / 2} \rightarrow{ }^{2} P_{1 / 2}$ fine-structure line of [C II] (a major cooling line of the ISM) is expected to be much brighter than the CO lines. It has long been known (e.g., Stacey et al. 1991; Malhotra et al. 1997) that [C II] is tracing star formation (photon-dominated regions, PDRs) and that it can carry up to $1 \%$ of the total far-infrared emission of a galaxy, in particular in systems of low luminosity and metallicity (e.g., Israel et al. 1996; Madden et al. 1997). The high ratio of $L_{[\text {C II] }} / L_{\text {FIR }}$ (see Section 3) is the reason why it has been argued for more than a decade that observation of the [C II] line of pristine systems at the highest redshifts will likely be the key to study the starforming ISM in the earliest star-forming systems (e.g., Stark 1997; Walter \& Carilli 2008; Carilli et al. 2008). Indeed, the last few years have seen a steep increase in the number of high- $z$ $(2<z<6.5)[\mathrm{C}$ II] detections, in most cases in systems that host an active galactic nucleus (AGN) and/or very high SFRs (»100 $M_{\odot} \mathrm{yr}^{-1}$; e.g., Maiolino et al. 2005, 2009; Walter et al. 2009; Stacey et al. 2010; Ivison et al. 2010; Wagg et al. 2010; Cox et al. 2011; De Breuck et al. 2011; Valtchanov et al. 2011).

[C II] is currently the most promising tracer of the starforming ISM (both PDRs and the cold neutral medium, CNM) in galaxies at the highest redshifts. However, a calibration to derive meaningful SFRs from $L_{\left[\mathrm{C}_{\text {II }}\right]}$ is still lacking. The [C II] line also traces the CNM and thus is a fundamental tracer for the overall distribution of the ISM and its global dynamics (and thus dynamical masses). In this paper, we present the results of a search for [C $\mathrm{II}]$ emission and the underlying FIR continuum in some of the highest redshift (non-AGN) galaxies with moderate UV-based SFR $\left(\sim 20 M_{\odot} \mathrm{yr}^{-1}\right)$. In Section 2, we describe the target selection and observations. The data are shown in Section 3 and implications are summarized in Section 4 . Throughout this paper we use a $\Lambda$-cold dark matter cosmology with $H_{0}=70 \mathrm{~km} \mathrm{~s}^{-1} \mathrm{Mpc}^{-1}, \Omega_{\Lambda}=0.7$, and $\Omega_{m}=0.3$.

\section{OBSERVATIONS}

\subsection{Source Selection}

\subsubsection{Ly Emitters}

Out of the many dozens of spectroscopically confirmed $z>6.5$ LAEs (see the caption to Figure 1 for references) we have selected two targets with high SFRs based on their Ly $\alpha$ and (rest-frame) UV continuum luminosity. The first source is an exceptionally luminous LAE at $z=6.595$ (Ouchi et al.

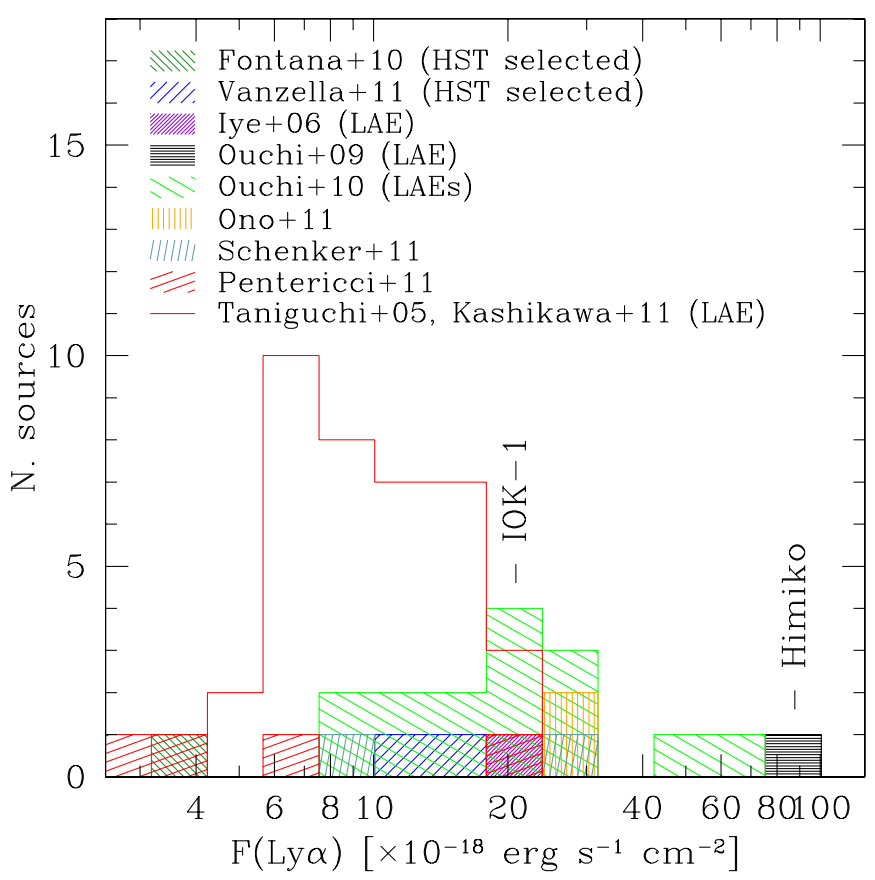

Figure 1. Distribution of Ly $\alpha$ fluxes (a proxy for star formation) in all published spectroscopically confirmed $z>6.5$ LAEs and LBGs (Taniguchi et al. 2005; Iye et al. 2006; Ouchi et al. 2009, 2010; Fontana et al. 2010; Vanzella et al. 2011; Kashikawa et al. 2011; Ono et al. 2012; Pentericci et al. 2011; Schenker \& Stark 2012). Most of these galaxies are too faint for detection in [C II] given current facilities. The targets selected in this study (Himiko and $I O K-1$ ) are among the brightest sources. $I O K-1$ is preferred over other LAEs of similar luminosity given its slightly higher redshift $(z \sim 7.0)$ and thus better receiver performance at the redshifted $[\mathrm{C}$ II] frequency.

2009) dubbed Himiko by these authors. Himiko is by far the brightest and most spatially extended $\left(\sim 3^{\prime \prime}\right)$ LAE known at these redshifts, with a lower limit to the SFR derived from the restframe UV emission (with no correction for dust attenuation) of $\sim 25 M_{\odot} \mathrm{yr}^{-1}$. Himiko is even detected at $3.6 \mu \mathrm{m}$ using Spitzer, implying a significant underlying stellar mass of $\sim 4 \times 10^{10} M_{\odot}$ (Ouchi et al. 2009).

The second source is $I O K-1$, an LAE at $z=6.96$ (Iye et al. 2006). This LAE is similar in both Ly $\alpha$ and continuum brightness to those in other samples at $z \sim 6.6$ (see Figure 1). The (rest-frame) UV emission implies an SFR of $\sim 16 M_{\odot} \mathrm{yr}^{-1}$ and HST imaging of $I O K-1$ gives a source size of $<1^{\prime \prime}$ (Cai et al. 2011).

\subsubsection{GRB 090423}

The third source is $G R B 090423$ (Krimm et al. 2009). VLT spectroscopy of GRB 090423 gave a redshift of $8.26_{-0.08}^{+0.07}$, based on a continuum break due to Gunn-Peterson absorption (Tanvir et al. 2009). A similar estimate was reported by Salvaterra et al. (2009) using spectroscopy at the TNG $\left(z=8.1_{-0.3}^{+0.1}\right)$. This makes GRB 090423 among the highest redshift GRBs discovered. The afterglow has been detected in the millimeter continuum by the IRAM Plateau de Bure Interferometer (PdBI) at $3 \mathrm{~mm}$ wavelengths with a flux density of $0.3 \pm 0.1 \mathrm{mJy}$ (Castro-Tirado et al. 2009; de Ugarte Postigo et al. 2012; obtained on 2009 April 23 and 24). An upper limit of the afterglow and the host galaxy FIR flux density at $1.2 \mathrm{~mm}$ wavelengths of $0.23 \pm 0.32 \mathrm{mJy}$ using MAMBO has also been reported (Riechers et al. 2009; obtained on 2009 April 25). 

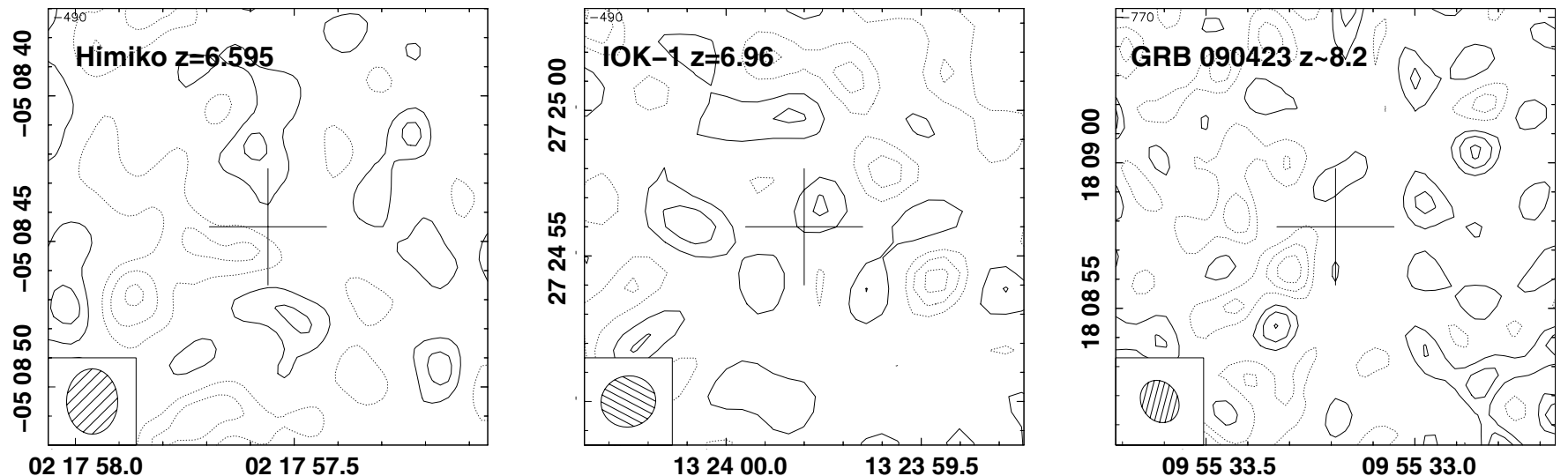

Figure 2. Rest-frame $158 \mu \mathrm{m}$ continuum observations of Himiko (left), IOK-1 (middle), and GRB 090423 (right panel). Coordinates are given in the J2000.0 system and contours are shown in steps of $1 \sigma$, starting at $\pm 1 \sigma\left(0.21 \mathrm{mJy} \mathrm{beam}^{-1}, 0.15 \mathrm{mJy} \mathrm{beam}^{-1}\right.$, and $0.089 \mathrm{mJy}^{\text {beam }}{ }^{-1}$, respectively, see Table 1$)$. No significant emission is detected from any of the sources.

Table 1

Summary of Observations and Derived Properties

\begin{tabular}{|c|c|c|c|c|c|c|c|c|c|c|}
\hline Source $^{a}$ & $\begin{array}{c}\text { R.A. } \\
\text { J2000.0 }\end{array}$ & $\begin{array}{c}\text { Decl. } \\
\text { J2000.0 }\end{array}$ & $z^{\mathrm{a}}$ & $\begin{array}{c}v^{\mathrm{b}} \\
(\mathrm{GHz})\end{array}$ & $\begin{array}{c}\sigma_{\text {cont }}{ }^{c} \\
\left(\mathrm{mJy} \mathrm{beam}^{-1}\right)\end{array}$ & $\begin{array}{c}\sigma_{\text {line }} \mathrm{d} \\
\left(\mathrm{mJy} \mathrm{beam}^{-1}\right)\end{array}$ & $\begin{array}{c}L_{[\mathrm{C} \text { II] }}^{\mathrm{e}} \\
\left(10^{8} L_{\odot}\right)\end{array}$ & $\begin{array}{c}L_{\mathrm{IR}}^{\mathrm{N} 6946} \\
\left(10^{11} L_{\odot}\right)\end{array}$ & $\begin{array}{l}\mathrm{SFR}^{\text {dust }>\mathrm{f}} \\
\left(M_{\odot} \mathrm{yr}^{-1}\right)\end{array}$ & $\begin{array}{l}\mathrm{SFR}^{\mathrm{UV}>\mathrm{g}} \\
\left(M_{\odot} \mathrm{yr}^{-1}\right)\end{array}$ \\
\hline Himiko & $02: 17: 57: 56$ & $-05: 08: 44.5$ & 6.595 & 250.361 & 0.21 & 0.70 & $<4.43$ & $<3.98$ & $<69$ & $\sim 25$ \\
\hline$I O K-1$ & $13: 23: 59.80$ & $+27: 24: 56.0$ & 6.96 & 238.881 & 0.15 & 0.65 & $<4.42$ & $<3.02$ & $<52$ & $\sim 16$ \\
\hline GRB 090423 & 09:55:33.19 & $+18: 08: 57.8$ & $\sim 8.2$ & $203.38,206.98$ & 0.089 & 0.61 & $<5.44$ & $<2.29$ & $<39$ & . \\
\hline
\end{tabular}

Notes. All luminosity upper limits are $3 \sigma$.

a References: Himiko: Ouchi et al. 2009; IOK-1: Iye et al. 2006; GRB 090423: Tanvir et al. 2009, Salvaterra et al. 2009.

${ }^{\mathrm{b}}$ Frequencies for Himiko and IOK-1 are tuned $\sim 125 \mathrm{MHz}$ blueward of Ly $\alpha$ redshift.

${ }^{\mathrm{c}} 1 \sigma$ continuum sensitivity at $158 \mu \mathrm{m}$ rest wavelengths.

d $1 \sigma\left[\mathrm{C}_{\mathrm{II}}\right]$ line sensitivity over a channel width of $200 \mathrm{~km} \mathrm{~s}^{-1}$.

e $3 \sigma$ [C II] luminosity limit over a channel width of $200 \mathrm{~km} \mathrm{~s}^{-1}$ assuming $L_{\text {line }}=1.04 \times 10^{-3} F_{\text {line }} v_{\text {rest }}(1+z)^{-1} D_{\mathrm{L}}^{2}$, where the line luminosity, $L_{\text {line }}$, is measured in $L_{\odot}$, the velocity-integrated flux, $F_{\text {line }}=S_{\text {line }} \Delta v$, in Jy km s ${ }^{-1}$, the rest frequency, $v_{\text {rest }}=v_{\text {obs }}(1+z)$, in GHz, and the luminosity distance, $D_{\mathrm{L}}$, in Mpc.

f $3 \sigma$ limit based on $L_{\mathrm{IR}}^{\mathrm{N} 6946}$.

g UV-based SFR from Ouchi et al. (2009) and Cai et al. (2011).

\subsection{PdBI Observations}

Himiko, IOK-1, and GRB 090423 have been observed with the IRAM Plateau de Bure interferometer using the WideX wide bandwidth correlator. The [C II] line (rest frequency: $1900.54 \mathrm{GHz}, 157.74 \mu \mathrm{m})$ is shifted to the $1 \mathrm{~mm}$ band and we have tuned the receivers $150 \mathrm{~km} \mathrm{~s}^{-1}(\sim 125 \mathrm{MHz})$ blueward of the redshift derived from optical spectroscopy (see Table 1 for the exact tuning frequencies). The total bandwidth of WideX is $3.6 \mathrm{GHz}$, or $\sim 4400 \mathrm{~km} \mathrm{~s}^{-1}$ at the observed frequencies. Thus any conceivable shift between the Ly $\alpha$ and the [C II] lines is covered by our observations. Observations were typically carried out during good observing conditions, using standard calibrations. $I O K-1$ was observed during three runs (2010 April-October) for an equivalent (five-element interferometer) on-source time of $9.1 \mathrm{hr}$ (Himiko: 7 days from 2010 August to December, total of $11.5 \mathrm{hr}$; GRB 090423: 4 days in 2010 April, total of $9.7 \mathrm{hr}$ ). The following gain calibrators were used-Himiko: B0336-019; IOK-1: B1308+326; GRB 090423: B1040+244.

Table 1 summarizes the sensitivity reached in the observations; here, we list the $1 \sigma$ continuum sensitivity as well as the $1 \sigma$ line sensitivity over a $200 \mathrm{~km} \mathrm{~s}^{-1}$ channel (which we adopt as realistic line widths of these sources, given their Ly $\alpha$ widths). The observations resulted in the following beamsizes: Himiko: $2^{\prime \prime} .27 \times 1^{\prime \prime} .73$, P.A. $=172^{\circ}(\mathrm{C}$ and D configurations $) ; I O K-1$ : $1^{\prime \prime} .88 \times 1^{\prime \prime} .75$, P.A. $=109^{\circ}$ (D configuration); and GRB 090423: $1^{\prime \prime} .51 \times 1^{\prime \prime} .22$, P.A. $=30.29$ (C configuration). Himiko has a spatial extent that is similar to our beamsize $\left(\sim 3^{\prime \prime}\right.$; Ouchi et al. 2009), so we consider the risk of outresolving the source as small.

\section{RESULTS AND DISCUSSION}

\subsection{Continuum Emission}

None of our sources is detected in the rest-frame FIR continuum (Figure 2). In Figure 3, we overplot our $3 \sigma$ continuum limit for Himiko, for which the best UV/optical data exist to date (Ouchi et al. 2009), on top of galaxy spectral energy distribution (SED) templates. The templates include Arp 220, M 82, M 51, and NGC 6946 (Silva et al. 1998), as well as spiral (cyan) and dwarf (blue) galaxy SEDs from the observations presented in Dale et al. (2007). All SEDs are shifted to Himiko's redshift $z=6.595$ and are normalized to the (rest-frame) UV continuum, shortly longward of the Ly $\alpha$ wavelength. Based on our continuum upper limit we can rule out a number of galaxy SED shapes. In particular, our measurement is (perhaps not surprisingly) incompatible with very obscured dusty starburst templates, such as Arp 220 and M 82, but also dust-rich nearby spiral 


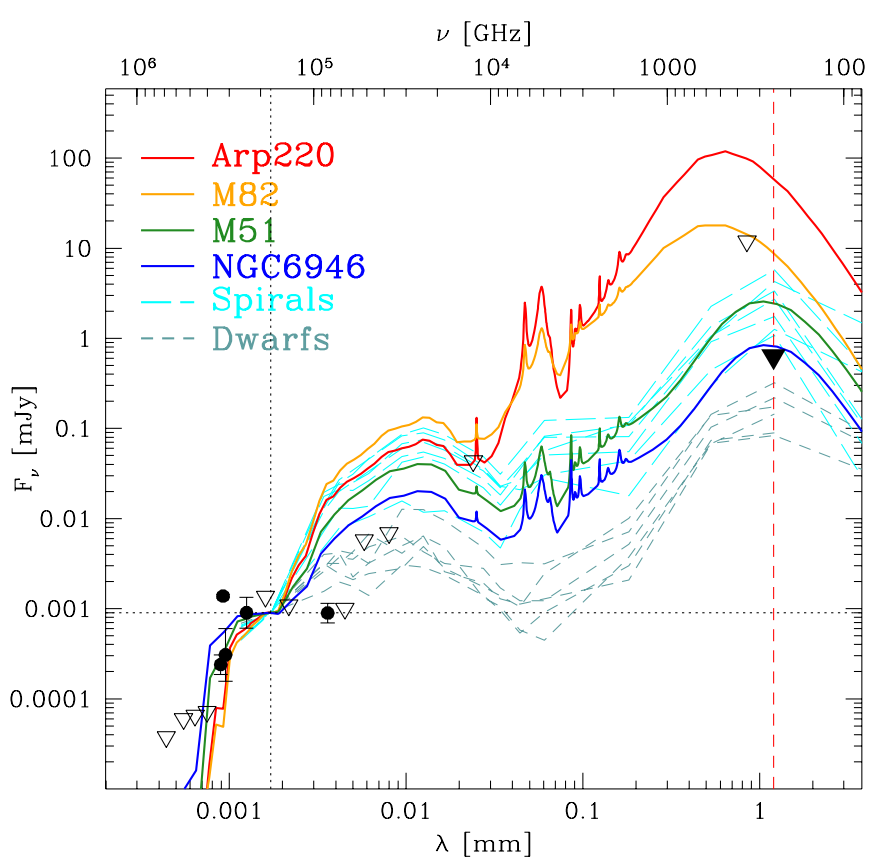

Figure 3. SED of Himiko in the observer's frame (bottom: wavelength, top: frequency). Detections in the (rest-frame) UV and $B$ band are indicated as black points (Ouchi et al. 2009). $3 \sigma$ upper limits from Ouchi et al. (2009) are shown as open triangles. Our $3 \sigma$ upper limit at $158 \mu \mathrm{m}$ (rest frame, see vertical red line) is shown as a black triangle. A number of galaxy SEDs are shown for comparison (see color coding in the panel) normalized to the UV measurement longward of $\operatorname{Ly} \alpha$ (as indicated by the vertical and horizontal dashed lines). The SEDs of Arp 220, M 82, M 51, and NGC 6946 are from Silva et al. (1998) and the cyan and gray curves show individual measurements of nearby spiral and dwarf galaxies, respectively (Dale et al. 2007). Our continuum limit rules out most spiral galaxy (and dustier system) SED shapes but is compatible with the SED shapes representative of lower dust content found in nearby dwarf galaxies.

galaxies such as M 51. For $I O K-1$ (not shown) the situation is very similar: the source has a similar redshift, millimeter continuum limit, and UV-based SFR (see Table 1).

Based on Figure 3 we would have detected the dust continuum at $>3 \sigma$ if Himiko's SED resembled M 51 or NGC 6946, but note that such SEDs (and those of more metal-poor dwarfs) are also already excluded from the IRAC $3.6 \mu \mathrm{m}$ measurement which corresponds to a rest wavelength of $475 \mathrm{~nm}$. This flux measurement, as discussed in detail in Ouchi et al. (2009), is dependent on the presumably young stellar component that is in place at $z=6.6$. As the relation of such a pristine stellar population to the dust emission, and the relative spatial distribution between the two, is unknown, the FIR limit is still relevant. In any case our observations point toward a dust-poor environment, e.g., as seen in the case of local dwarf galaxies (see dwarf galaxy SEDs in Figure 3).

Figure 3 shows that our continuum measurement is close in wavelength to the peak of any likely galaxy SED (spiral/dwarfs). In the following, we derive a conservative upper limit for $L_{\mathrm{IR}}$ (integrated from 8 to $1000 \mu \mathrm{m}$ ) using the galaxy template for NGC 6946, scaled to our $3 \sigma$ upper limit (we consider this limit conservative as using a dwarf galaxy template would decrease this upper limit). From this we derive $3 \sigma$ upper limits of the (F)IR-based SFR (using $\operatorname{SFR}\left(M_{\odot} \mathrm{yr}^{-1}\right)=1.72 \times$ $10^{-10} L_{\mathrm{IR}}\left(L_{\odot}\right)$, Kennicutt 1998) of $\sim 70 \mathrm{M}_{\odot} \mathrm{yr}^{-1}$ (Himiko) and $\sim 50 M_{\odot} \mathrm{yr}^{-1}$ (IOK-1, see Table 1$)$. We note that our upper limit of the FIR-based SFR for Himiko and $I O K-1$ is only a factor $\sim 3$ higher than the UV-based SFR (not corrected for extinction).

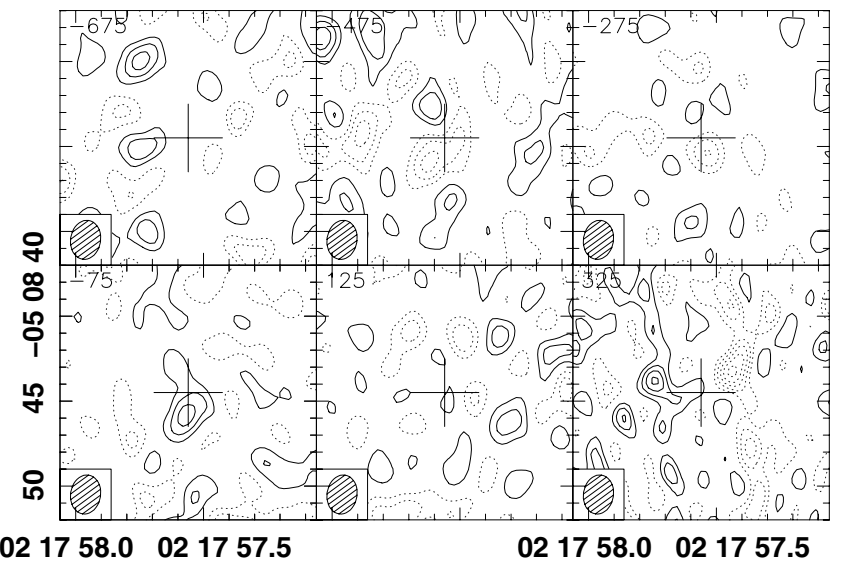

Figure 4. Himiko [C II] velocity channel maps (width: $200 \mathrm{~km} \mathrm{~s}^{-1}$ ) around the expected redshift. Contours are shown in steps of $1 \sigma\left(0.70 \mathrm{mJy}^{\mathrm{beam}}{ }^{-1}\right)$ starting at $\pm 1 \sigma$. Central velocities are shown in the top left corner of each panel. Note that due to our tuning the systemic redshift corresponds to a velocity of $-150 \mathrm{~km} \mathrm{~s}^{-1}$ in this representation (see Section 2.2). A tentative $3 \sigma$ signal is seen $\sim 1^{\prime \prime} .5$ away from the phase center in the bottom left panel.

In the case of the $G R B 090423$ host galaxy we derive an upper limit of its FIR-based SFR $<39 M_{\odot} \mathrm{yr}^{-1}$ using the same SED template as above. Our SFR limits for GRB 090423 are the deepest FIR-based SFR measurement of a GRB to date (see, e.g., Berger et al. 2003; de Ugarte Postigo et al. 2012; Hatsukade et al. 2011). Very recent deep HST observations did not reveal the host galaxy of $G R B 090423$ either down to a limiting UV-based SFR of $0.4 M_{\odot} \mathrm{yr}^{-1}$ (Tanvir et al. 2012).

\section{2. [C II] Line}

As in the case of the continuum, no significant [C II] emission is detected in our observations (see limits in Table 1). In Figure 4, we show the channel maps around the expected Ly $\alpha$ redshift for Himiko. We note a tentative $(3 \sigma)$ signal $1^{\prime \prime} .5\left(0.7 \times \mathrm{FWHM}_{\text {maj }}\right)$ south of the phase center, close to the central tuning frequency, but more sensitive observations are needed to further investigate this finding.

In Figure 5, we plot the ratio $L_{[\mathrm{C}}{ }_{\mathrm{II}} / L_{\mathrm{FIR}}$ as a function of $L_{\mathrm{FIR}}$ for objects taken from the literature (see references in the caption). Given our limits on the FIR and [C II] luminosities we can now constrain the location of our targets in this plot. The likely region in parameter space is shown as a shaded area in Figure 5 for Himiko (the limits for $I O K-1$ are very similar and are thus not shown for clarity). With an upper limit to $L_{\mathrm{FIR}}=$ $\sim 4 \times 10^{11} L_{\odot}$ our objects are the least FIR-bright sources (by an order of magnitude) observed at high redshift and start to probe the region that is only covered by local galaxies at present.

We have shown above that the FIR-based SFRs cannot be significantly larger than the UV-based ones. If we assume that the FIR-based SFR in these objects would equal the UV-based SFR, we can assign a hypothetical FIR luminosity to both Himiko and $I O K-1$ (this luminosity would be lower by a factor of a few if we had assumed a dwarf SED template, see Figure 3). This results in the (upper limit) data points shown in Figure 5. Under this assumption we would thus be able to rule out high ratios such as $L_{[\mathrm{C} \text { II] }} / L_{\mathrm{FIR}} \sim 0.01$ found in low-metallicity and low-luminosity nearby galaxies (e.g., Israel et al. 1996; Madden et al. 1997). We stress, however, that we have few constraints on $L_{\mathrm{FIR}}$ for both sources, e.g., a very low $L_{\mathrm{FIR}}$ could result in significantly higher $L_{[\mathrm{CI}} / L_{\mathrm{FIR}}$ ratios. 


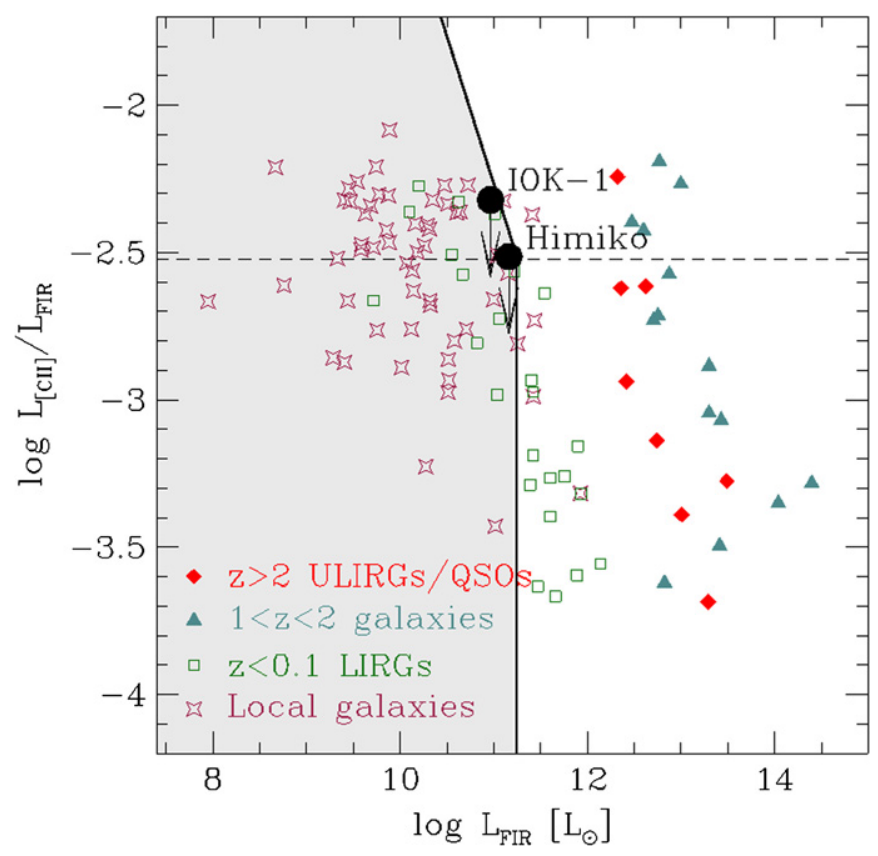

Figure 5. $L_{[\mathrm{CII}} / L_{\mathrm{FIR}}$ as a function of $L_{\mathrm{FIR}}$ for published [C II] detections (Malhotra et al. 2001; Maiolino et al. 2009; Stacey et al. 2010; Ivison et al. 2010; Wagg et al. 2010; Cox et al. 2011; De Breuck et al. 2011). As neither FIR nor [C II] emission is detected in our targets, the shaded area indicates the possible parameter for Himiko and $I O K-1$ based on the upper limits. Assuming that the FIR-based (extincted) SFR equals the UV-based (unextincted) SFR (i.e., an FIR luminosity that is three times lower than our upper limit) results in the two upper limit datapoints shown for Himiko and $I O K-1$. The horizontal dashed line indicates the average value of $L_{[\mathrm{C} \text { II] }} / L_{\mathrm{FIR}}=3 \times 10^{-3}$ for local galaxies.

\section{CONCLUDING REMARKS}

We have presented the results of a search for the $[\mathrm{C} \mathrm{II}]^{2} P_{3 / 2} \rightarrow$ ${ }^{2} P_{1 / 2}$ line and the underlying continuum emission at $158 \mu \mathrm{m}$ in two LAEs at high redshifts that form stars at moderate rates (UV-based SFR $\sim 20 M_{\odot} \mathrm{yr}^{-1}$ ), and a $z \sim 8.2$ GRB. Our sources are the first to push studies of (unlensed) high- $z$ sources to FIR luminosities less than a few times $10^{11} L_{\odot}$ and the resulting [C II]/FIR limits begin to probe the luminosities and parameter space occupied by local star-forming galaxies. The continuum measurements in the LAEs rule out galaxy SEDs that show extreme extinction, such as Arp 220 and M 82, and even those of dust-rich nearby spiral galaxies such as M 51. Based on our $L_{\mathrm{FIR}}$ limits we derive that the (obscured) SFR cannot be higher than a factor of $\sim 3$ times based on the UV-derived SFR. This correction factor (to account for obscured star formation) is lower than the typical correction factor of $\sim 5$ to correct UV-based SFRs for extinction in lower-redshift Lyman break galaxies (LBGs; e.g., discussion in Pannella et al. 2009). Our correction factor is also significantly lower than the one derived for the Herschel PACS-detected subsample of LAEs at $2.0<z<3.5$ where the total SFRs exceed the UV-based ones by more than an order of magnitude (Oteo et al. 2012b, their Table 1). However, our finding is consistent with recent (restframe) UV studies of $z \sim 7$ LBGs that also show only very little evidence for extinction in systems of very similar redshifts (e.g., Bouwens et al. 2010).

Observations of the [C II] line in galaxies at such high redshifts as probed here will play a critical role in the future (e.g., Walter \& Carilli 2008): the brightest line in the NIR (Ly $\alpha$ ) is highly resonant and will be affected by absorption in the intergalactic medium (Gunn-Peterson effect) as well as possible dust aborption within the galaxy. [C II] emission, on the other hand, can in principle provide key information on the star formation properties and dynamical masses at these redshifts. The increase in sensitivity afforded by ALMA will push the SFR limits obtained from [C II] and FIR continuum measurements down to only a few $M_{\odot} \mathrm{yr}^{-1}$. We note, however, that the lack of a sufficient number of ALMA "band 5" receivers (163-211 GHz) will not allow one to easily study galaxies emerging from the Epoch of Reionization in the redshift range $8.0<z<10.65$ with ALMA.

We thank the referee for critical and constructive comments that helped to improve this paper. We thank Dale Frail and Ruben Salvaterra for useful discussions concerning GRB host galaxies. Based on observations with the IRAM Plateau de Bure Interferometer. IRAM is supported by INSU/CNRS (France), MPG (Germany), and IGN (Spain). R.D. is supported by DLR grant FKZ-50-OR-1104. D.R. acknowledges funding through a Spitzer Space Telescope grant. We acknowledge the use of GILDAS software (http://www.iram.fr/IRAMFR/GILDAS).

\section{REFERENCES}

Berger, E., Cowie, L. L., Kulkarni, S. R., et al. 2003, ApJ, 588, 99 Bouwens, R. J., Illingworth, G. D., Labbe, I., et al. 2011, Nature, 469, 504 Bouwens, R., Illingworth, G. D., Oesch, P. A., et al. 2010, ApJ, 708, L69 Bromm, V., \& Loeb, A. 2006, ApJ, 642, 382

Cai, Z., Fan, X., Jiang, L., et al. 2011, ApJ, 736, L28

Carilli, C., Walter, F., Wang, R., et al. 2008, Ap\&SS, 313, 307

Castro-Tirado, A. J., Bremer, M., Winters, J.-M., et al. 2009, GCN Circ., 9273, 1

Cox, P., Krips, M., Neri, R., et al. 2011, ApJ, 740, 63

Dale, D. A., Gil de Paz, A., Gordon, K. D., et al. 2007, ApJ, 655, 863

De Breuck, C., Maiolino, R., Caselli, P., et al. 2011, A\&A, 530, L8

de Ugarte Postigo, A., Lundgren, A., Martín, S., et al. 2012, A\&A, 538, A44

Fan, X., Carilli, C. L., \& Keating, B. 2006, ARA\&A, 44, 415

Fontana, A., Vanzella, E., Pentericci, L, et al. 2010, ApJ, 725, L205

Hatsukade, B., Kohno, K., Endo, A., Nakanishi, K., \& Ohta, K. 2011, ApJ, 738, 33

Israel, F. P., Maloney, P. R., Geis, N., et al. 1996, ApJ, 465, 738 Ivison, R., Swinbank, A. M., Swinyard, B., et al. 2010, A\&A, 518, L35 Iye, M., Ota, K., Kashikawa, N., et al. 2006, Nature, 443, 186 Jiang, L., Egami, E., Kashikawa, N., et al. 2011, ApJ, 743, 65 Kashikawa, N., Shimasaku, K., Matsuda, Y., et al. 2011, ApJ, 734, 119 Kennicutt, R. C. 1998, ARA\&A, 36, 189

Kistler, M. D., Yüksel, H., Beacom, J. F., \& Stanek, K. Z. 2008, ApJ, 673, L119

Krimm, H. A., Beardmore, A. P., Evans, P. A., et al. 2009, GCN Circ., 9198, 1 Madden, S. C., Poglitsch, A., Geis, N., Stacey, G. J., \& Townes, 1997, ApJ, 483, 200

Maiolino, R., Caselli, P., Nagao, T., et al. 2009, A\&A, 500, L1

Maiolino, R., Cox, P., Caselli, P., et al. 2005, A\&A, 440, L51

Malhotra, S., Helou, G., Stacey, G., et al. 1997, ApJ, 491, L27

Malhotra, S., Kaufman, M. J., Hollenbach, D., et al. 2001, ApJ, 561, 766

Ono, Y., Ouchi, M., Mobasher, B., et al. 2012, ApJ, 744, 83

Oteo, I., Bongiovanni, A., Pérez García, A. M., et al. 2012a, arXiv:1202.0782

Oteo, I., Bongiovanni, A., Pérez García, A. M., et al. 2012b, A\&A, 541, A65

Ouchi, M., Ono, Y., Egami, E., et al. 2009, ApJ, 696, 1164

Ouchi, M., Shimasaku, K., Furusawa, H., et al. 2010, ApJ, 723, 869

Pannella, M., Carilli, C. L., Daddi, E., et al. 2009, ApJ, 698, L116

Pentericci, L., Fontana, A., Vanzella, E., et al. 2011, ApJ, 743, 132

Riechers, D. A., Walter, F., Bertoldi, F., et al. 2009, GCN Circ., 9322, 1

Robertson, B., Ellis, R. S., Dunlop, J. S., McLure, R. J., \& Stark, D. P. 2010, Nature, 468, 49

Salvaterra, R., \& Chincarini, G. 2007, ApJ, 656, L49

Salvaterra, R., Della Valle, M., Campana, S, et al. 2009, Nature, 461, 1258

Schenker, M. A., Stark, D. P., et al. 2012, ApJ, 744, 179

Silva, L., Granato, G. L., Bressan, A., \& Danese, L. 1998, ApJ, 509, 103

Solomon, P. M., \& Vanden Bout, P. A. 2005, ARA\&A, 43, 677

Stacey, G. J., Geis, N., Genzel, R., et al. 1991, ApJ, 373, 423

Stacey, G. J., Hailey-Dunsheath, S., Ferkinhoff, C., et al. 2010, ApJ, 724, 957

Stark, A. A. 1997, ApJ, 481, 587 
Taniguchi, Y., Ajiki, M., Nagao, T., et al. 2005, PASJ, 57, 165

Tanvir, N. R., Fox, D. B., Levan, A. J., et al. 2009, Nature, 461, 1254

Tanvir, N. R., Levan, A. J., Fruchter, A. S., et al. 2012, ApJ, submitted (arXiv:1201.6074)

Valtchanov, I., Virdee, J., Ivison, R. J., et al. 2011, MNRAS, 415, 3473

Vanzella, E., Pentericci, L., Fontana, A., et al. 2011, ApJ, 730, L35
Wagg, J., Carilli, C. L., Wilner, D. J., et al. 2010, A\&A, 519, L1

Walter, F., \& Carilli, C. 2008, Ap\&SS, 313, 313

Walter, F., Carilli, C., \& Daddi, E. 2011, arXiv:1101.4022

Walter, F., Riechers, D., Cox, P., et al. 2009, Nature, 457, 699

Woosley, S. E., \& Bloom, J. S. 2006, ARA\&A, 44, 507

Yüksel, H., Kistler, M. D., Beacom, J. F., \& Hopkins, A. M. 2008, ApJ, 683, L5 